\title{
Output Current Ripple Factor Performance of Half-Wave Rectifier With and Without Freewheeling Diode
}

\author{
Taufik \\ Ali O. Shaban \\ Ahmad Nafisi \\ Department of Electrical Engineering \\ California Polytechnic State University \\ San Luis Obispo, CA 93407
}

\begin{abstract}
The objective of this work is to investigate ripple factor of half-wave rectifier circuits. The ripple factor is one of important characteristic necessary when designing a power electronic converter. The ripple factor measures how much deviation the converter output parameter has, such as the output current, from its nominal designed value. In this paper the ripple factor of the output current of half-wave rectifiers will be investigated. More specifically, the ripple factor of output current from more practical half-wave rectifiers that include inductive load with or without a freewheeling diode will be analyzed and then compared to that of the basic half-wave rectifier consisting only of a resistive load. Derivation of the equations for the ripple factor for the three half-wave rectifier circuits will first be presented. From these results, plots will be generated using Pspice that will allow us to conveniently compare the ripple factor performance of each of the rectifiers.
\end{abstract}

\section{INTRODUCTION}

In power conversion circuits, the actual output waveform of any converter constructed from the input sources will in general be different from the input. This tells us that the output must contain unwanted components along with the wanted components. These unwanted components are unfortunately unavoidable and they can be described as the Fourier components. The complete collection of unwanted components defines distortion. The terms harmonic distortion or simply harmonics refer to this unwanted behavior. Particularly in dc application such as that of rectifier circuits, the collected unwanted components are typically referred to by the term ripple. A more specific definition is ripple factor which is a tool to measure how much deviation the converter output parameter has from its nominal designed value.

Due to the fact that ripple is closely related to distortion of the output waveform, it is therefore crucial to consider addressing the questions about distortions in the initial steps of converter design. How much the load can tolerate, whether or not there are specific frequencies that are especially bad and should be avoided, the necessity for filtering, and specifying distortion limits are some of the ripple-related issues that might be brought up prior to designing the converter. Moreover, prior knowledge of the ripple factor is also very significant since it can be used as an indicator of the quality of the output waveform. From practical point of view, knowledge of the ripple factor before hand will aid us in designing a cost-effective converter sircuit, such determining the need of having an extra freewheeling diode used in a halfwave rectifier to improve the quality of output waveform.

In most dc applications, the output ripple represents both a variation around the desired dc level and a possible energy effect at undesired frequencies [1]. In a standard dc supply, it is common to specify the maximum peak to peak ripple, and often the ripple rms magnitude as well. For example, typical numbers for peak to peak ripple fall in the 50-100 mv range for low dc voltages. A good rule of thumb is that the ripple will be about $1 \%$ of the nominal output.

\section{THE BASIC HALF-WAVE RECTIFIER}

Rectification is the process of converting ac power to dc power. An uncontrolled rectifier uses only diodes as rectifying elements. The dc output voltage is fixed in magnitude by the amplitude of the ac supply voltage. However, the dc output is not pure, that is, it contains significant ac components or ripple. To suppress this ripple, a filter is typically inserted after the rectifier. The simplest circuit configuration of rectifier is the half-wave rectifier and is shown in figure 1 . Here, the half-wave rectifier circuit whose source voltage is a sine wave is supplying a purely resistive load. During the positive half cycle, when the voltage at the anode is positive with respect to the cathode, the diode turns on. This allows current through the load resistor. Thus, the load voltage follows the positive half sine wave. During the negative half-cycle, the voltage at the anode becomes negative with respect to the cathode and the diode turns off. There is no current flows through $R$. Figure 2 shows the output voltage waveform of the half-wave rectifier using an input waveform of $\hat{V}_{s} \sin \omega t$ and with frequency of $60 \mathrm{~Hz}$.

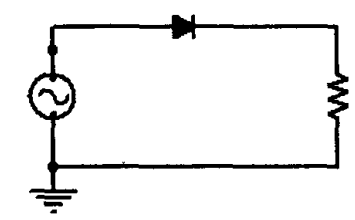

Fig. 1. Half-wave rectifier with resistive load 


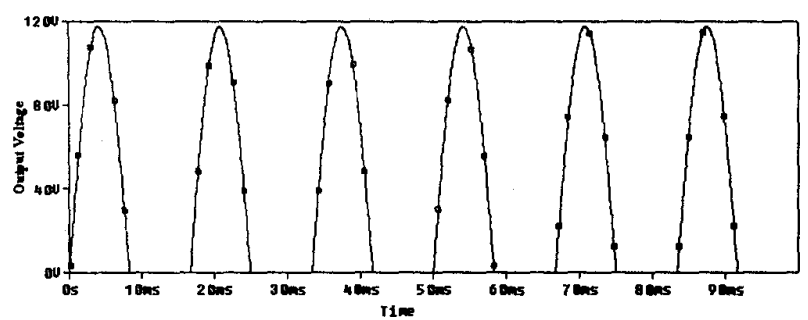

Fig. 2. Load voltage waveform of half-wave rectifier with resistive load

The load current, $I_{R}$, which is equal to the diode current will be periodic since the input signal is periodic. However, similar to the output voltage, the load current is also a very poor dc with its value equal to its average:

$I_{R}=\frac{1}{2 \pi} \int_{\alpha}^{2 \pi+\alpha} i_{R}(t) d \omega t=\frac{1}{2 \pi} \frac{\hat{V_{s}}}{R} \int_{0}^{\pi} \sin \omega t d \omega t=\frac{\hat{V_{s}}}{\pi R}$

Equation (1) shows that with this type of rectifier the average load current is less than $1 / 3$ of its peak value.

\section{THE RIPPLE FACTOR}

Using the previous example, we can easily see that the load current will have ripple, and if we define this ripple to be:

$I_{R r}=\sqrt{\left|I_{R}\right|^{2}-I_{R}^{2}}$

where:

$\left|I_{R}\right|=\sqrt{\frac{1}{2 \pi} \int_{\alpha}^{2 \pi+\alpha} i_{R}^{2}(t) d \omega t}$

is the rms value of the load current, then we can define the normalized ripple or ripple factor which is given by;

$K_{r}=\frac{I_{R r}}{I_{R}}=\sqrt{\left(\frac{\left|I_{R}\right|}{I_{R}}\right)^{2}-1}$

The ripple factor is dimensionless and is useful in measuring how much deviation the output parameter has from its nominal designed value, and therefore plays an important role in designing a converter circuit.

To determine the ripple factor of our previous example, the rms value of the load current needs to be calculated:

$\left|I_{R}\right|=\sqrt{\frac{1}{2 \pi} \int_{0}^{\pi} \frac{\hat{V}_{s}^{2}}{R^{2}} \sin ^{2} \omega t d \omega t}=\frac{\hat{V_{s}}}{2 R}$
Substitutions of equations (1) and (5) into equation (4) yields:

$\mathrm{K}_{\mathrm{r}}=1.21$

which tells us that the ripple factor for a half-wave rectifier with resistive load is constant.

\section{THE HALF-WAVE RECTIFIER WITH INDUCTIVE LOAD}

An improved version of the basic half-wave rectifier circuit is depicted in figure 3, whereby an inductive load is connected rather than just a simple resistive load.

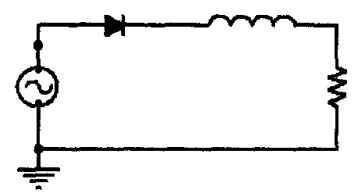

Fig. 3. Half-wave rectifier with inductive load

In steady state, the output current $I_{R}(t)$ must be periodic since the sinusoidal input waveform is a periodic waveform. By applying KVL:

$\frac{d i_{R}(t)}{d t}+\frac{R}{L} i_{R}(t)=\frac{\hat{V}}{L} \sin \omega t$

It can be shown that the solution for the this differential equation is:

$i_{R}(t)=\frac{\hat{V}_{s}}{\sqrt{R^{2}+\omega^{2} L^{2}}}\left[\sin \phi e^{-\frac{R}{L} t}+\sin (\omega t-\phi)\right]$

where:

$\phi=\tan ^{-1}\left[\frac{\omega L}{R}\right]$

Using the same method as was done in equation (1), the average value of the current described by equation (8) with conduction angle $\alpha$ is found to be:

$I_{R}=\frac{\hat{V}_{s}}{\sqrt{R^{2}+\omega^{2} L^{2}}} \frac{L}{R} \sin \phi\left[1-e^{-\frac{R \alpha}{L \omega}}\right]$

The rms of equation (8) can be derived by using the same definition in equation (5) which yields to:

$\left|I_{R}\right|=0.564 A \frac{\hat{V}_{s}}{\sqrt{R^{2}+\omega^{2} L^{2}}} \frac{L}{R} \sin \phi$ 
where:

$$
A=\sqrt{\left(1-e^{-\frac{2 R}{L}}\right)+\frac{\left(1-e^{-\frac{1}{L}}\right)}{\sin \phi}+\frac{R}{4 L \sin ^{2} \phi}\left[(\alpha-\phi)-\frac{1}{2} \sin 2(\alpha-\phi)\right]}
$$

As previously done in the case of the purely resistive load, once the expressions for the average value and the rms are obtained, the ripple factor may then be derived using (4).

A more practical half wave rectifier is illustrated in figure 4. Here the insertion of an extra diode allows the load current to continue to flow, or in other words to freewheel the current. Henceforth, this additional diode is called the freewheeling diode. For continuous conduction, that is the current never decays to zero, it can be shown that the ratio of the inductor and the load resistor has to be greater than $\pi / \omega$.

The load current for this particular circuit can be described as:

$$
i_{R}(t)=\frac{\hat{V} s}{Z}\left[\sin (\omega t-\phi)+\frac{\sin \phi}{1-e^{-\frac{R}{2 L} t}} e^{-\frac{R}{L} t}\right]
$$

when $0<\omega t<\pi$

And,

$i_{R}(t)=\frac{\hat{V}}{Z}\left[\frac{\sin \phi}{1-e^{-\frac{R}{2 L} t}} e^{-\frac{R}{L}\left(\frac{T}{2}-t\right)}\right]$

where $\pi<\omega t<2 \pi$

with:

$Z=\sqrt{R^{2}+(\omega L)^{2}}$

The same procedure can again be conducted to yield the average and rms values of the currents in (13) and (14), as were done in the previous two cases using the same definitions as shown in (3) and (5) respectively. Once these values are obtained, then the ripple factor equation for these currents can be derived using equation (4).

\section{COMPUTER SIMULATIONS}

To aid the analysis of the ripple factors of the three cases, namely, the half-wave rectifier with resistive load, with inductive load, and with freewheeling diode, a computer simulation using Pspiçe is used. Using this tool, we will be

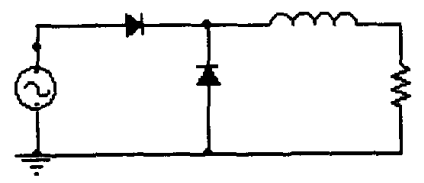

Fig. 4. Half-wave rectifier with inductive load able to investigate which scheme yields the smallest ripple factor. Recall that for the case where the half-wave rectifier has resistive load only, the ripple factor is found to be constant as shown in (6).

The three different circuit configurations for the half-wave rectifier, as shown in figures 1,3 , and 4 are then simulated and the results are recorded and compiled to produce several plots from which we can conveniently check to see which configuration gives us the best performance in terms of the ripple factor. Overall, five different cases are provided using different values of either the resistor load or the inductor. The first three cases, the value of the recistor is kept constant at $R=0.1 \Omega, 1 \Omega$, and $10 \Omega$ for cases 1,2 , and 3 respectively. In the last two cases, the inductor values are fixed at $\mathrm{L}=30 \mathrm{mH}$ and $300 \mathrm{mH}$ for cases 4 and 5 respectively. The results of the computer simulations for all five cases are shown in figures $5,6,7,8$ and 9 .

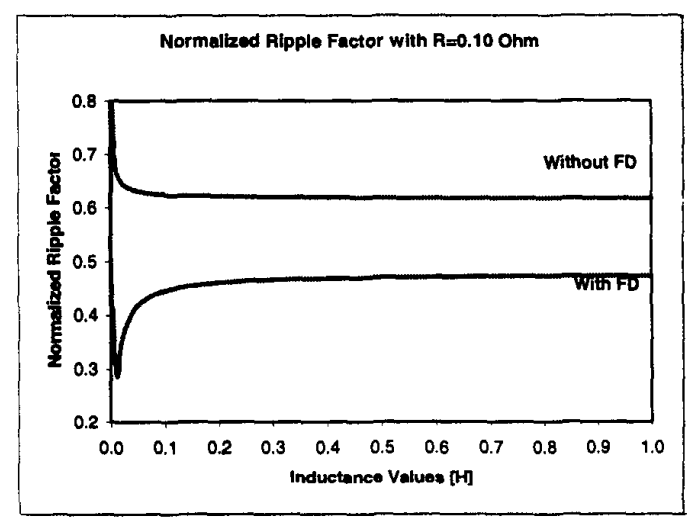

Fig. 5. Ripple factor with and without Freewheeling Diode (FD) when $R=0.10 \Omega$ - Case 1

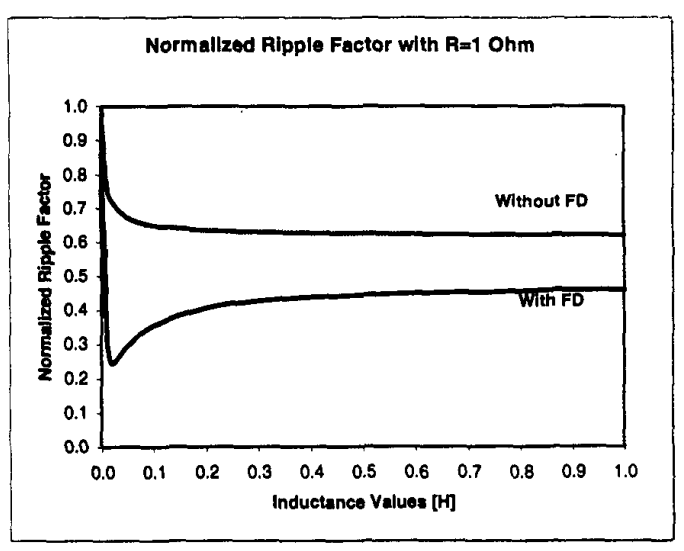

Fig. 6. Ripple Factor with and without Freewheeling Diode (FD) when $R=1 \Omega$ - Case 2 


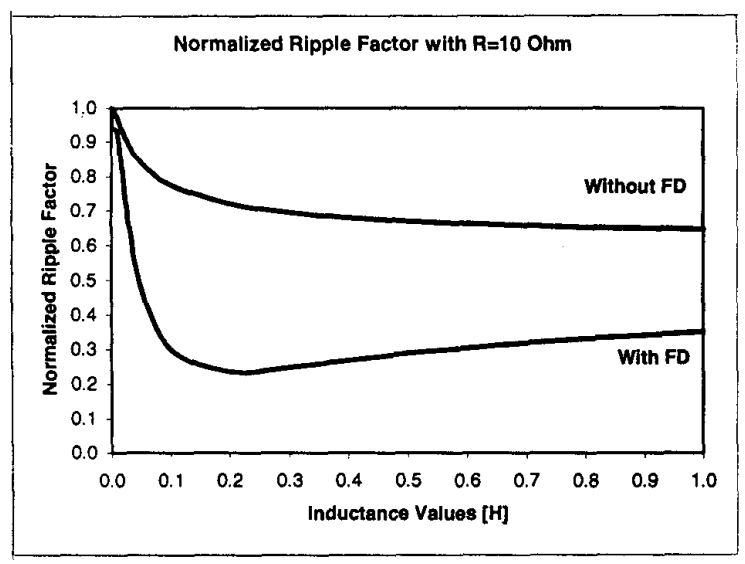

Fig. 7. Ripple Factor with and without Freewheeling Diode (FD) when $R=10 \Omega$ - Case 3

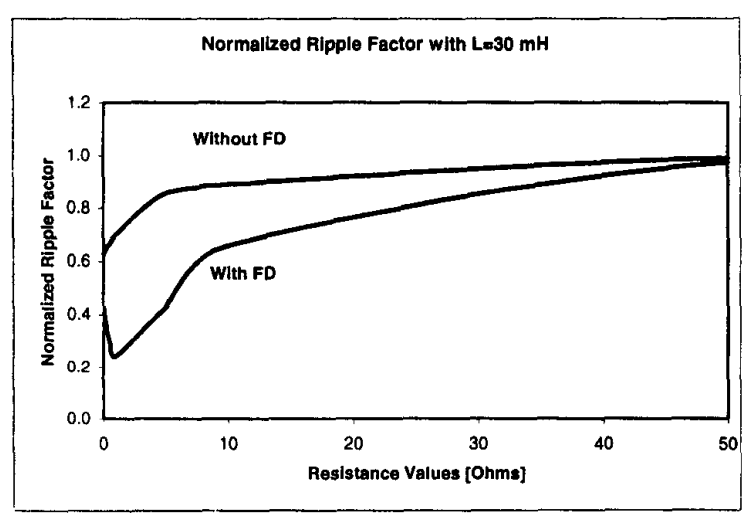

Fig. 8. Ripple Factor with and without Freewheeling Diode (FD) when $\mathrm{L}=30 \mathrm{mH}$ - Case 4

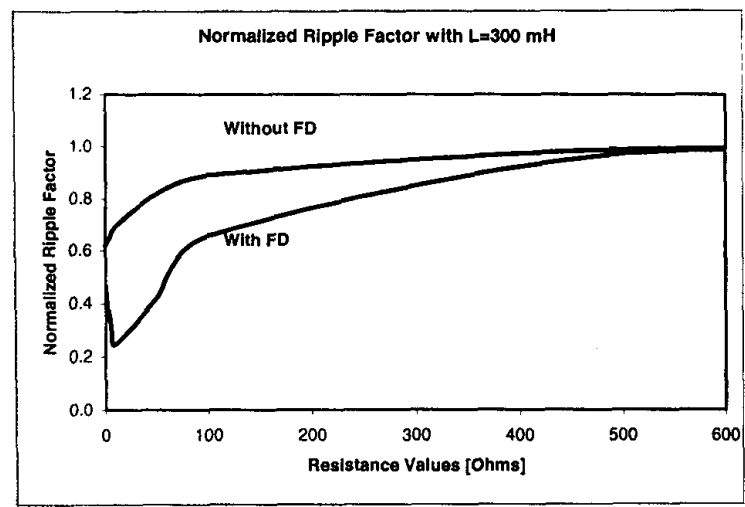

Fig. 9. Ripple Factor with and without Freewheeling Diode (FD) when $\mathrm{L}=300 \mathrm{mH}$ - Case 5
The results show that in general the half-wave rectifier with freewheeling diode performs the best in terms of the ripple factor of its output current. In the case of half-wave rectifier with resistive load, the value of the ripple factor is constant at 1.21 and is independent of the load resistor and the amplitude of the input voltage source. However, as the results further show, this is not true for the other two rectifiers with inductive load where the ripple factor is dependent upon both the load impedance and the input source. In the half-wave rectifier with freewheeling diode, if the load resistor is kept constant while inductance value is varied, the ripple factor of half-wave rectifier with freewheeling diode is always lower than those of the other two rectifiers. Moreover, its minimum ripple factor is found to be about 0.25 . Another interesting observation is that the higher the value of the load resistor, the higher the value of the inductance needed to reach its minimum ripple factor point. In another case where the inductance is fixed but the load resistor is varied, the ripple factor for both half-wave rectifiers with inductive load will reach its lowest value and then converges to the ripple factor of the half-wave rectifier with resistive load.

\section{CONCLUSIONS}

In this paper, the ripple factor characteristics of three different circuit configurations of the half-wave rectifiers were investigated. Several examples on how to derive the equations for the average and rms values were presented from which the expression for the ripple factor can further be obtained. Results from computer simulations were provided and several plots were included from five different cases. From these results, it is clear that the smallest and therefore the best ripple factor can be obtained when inductive load with the extra freewheeling diode is used. Further work for this project will include, but not limited to, lab implementations and measurements of these circuits to supply us with empirical results which can then be compared with those results that are obtained from the computer simulations.

\section{REFERENCES}

[1] P.T. Krein, Elements of Power Electronics, $1^{\text {st }}$ ed., Oxford, New York, 1998. 